\title{
Interfacing and Verifying ALHAT Safe Precision Landing Systems with the Morpheus Vehicle
}

\author{
John M. Carson III ${ }^{1,2, *}$, Robert L. Hirsh ${ }^{1, \dagger}$ Vincent E. Roback ${ }^{3, \ddagger}$, \\ Carlos Y. Villalpando ${ }^{2, \S}$, Joseph L. Busa ${ }^{4,}$ Diego F. Pierrottet ${ }^{5, \|}$ \\ Nikolas Trawny ${ }^{2, * *}$ Keith E. Martin ${ }^{2, \dagger \dagger}$ Glenn D. Hines ${ }^{3, \ddagger \ddagger}$ \\ ${ }^{1}$ NASA Johnson Space Center, ${ }^{2}$ Jet Propulsion Laboratory, California Institute of Technology, \\ ${ }^{3}$ NASA Langley Research Center, ${ }^{4}$ Helios Solutions, LLC., ${ }^{5}$ Coherent Applications, Inc.
}

\begin{abstract}
The NASA Autonomous precision Landing and Hazard Avoidance Technology (ALHAT) project developed a suite of prototype sensors to enable autonomous and safe precision landing of robotic or crewed vehicles under any terrain lighting conditions. Development of the ALHAT sensor suite was a cross-NASA effort, culminating in integration and testing onboard a variety of terrestrial vehicles toward infusion into future spaceflight applications. Terrestrial tests were conducted on specialized test gantries, moving trucks, helicopter flights, and a flight test onboard the NASA Morpheus free-flying, rocket-propulsive flighttest vehicle. To accomplish these tests, a tedious integration process was developed and followed, which included both command and telemetry interfacing, as well as sensor alignment and calibration verification to ensure valid test data to analyze ALHAT and Guidance, Navigation and Control (GNC) performance. This was especially true for the flight test campaign of ALHAT onboard Morpheus. For interfacing of ALHAT sensors to the Morpheus flight system, an adaptable command and telemetry architecture was developed to allow for the evolution of per-sensor Interface Control Design/Documents (ICD $)$. Additionally, individual-sensor and on-vehicle verification testing was developed to ensure functional operation of the ALHAT sensors onboard the vehicle, as well as precision-measurement validity for each ALHAT sensor when integrated within the Morpheus GNC system. This paper provides some insight into the interface development and the integrated-systems verification that were a part of the build-up toward success of the ALHAT and Morpheus flight test campaigns in 2014. These campaigns provided valuable performance data that is refining the path toward spaceflight infusion of the ALHAT sensor suite.
\end{abstract}

\section{Introduction}

The NASA ALHAT project has developed and terrestrially flight tested a suite of prototype GNC technologies (sensors and algorithms) applicable to crewed and robotic vehicles that enable autonomous and safe precision landing onto the lunar surface under any terrain lighting conditions $112[3$ The prototype sensor suite was tested in 2014 onboard the NASA Morpheus rocket-propulsive, terrestrial flight-test vehicle, or

\footnotetext{
*ALHAT SE\&I Lead, IPA Detail to JSC from JPL, AIAA Associate Fellow.

$\dagger$ ALHAT/Morpheus Ground Software Lead.

¥ALHAT Flash Lidar Systems Engineer, AIAA Senior Member.

$\S$ ALHAT HDS Software and Computing Lead.

\Morpheus Software Development.

${ }$ ALHAT NDL and LAlt Systems Engineer, AIAA Member.

**ALHAT HDS Lead, AIAA Member.

${ }^{\dagger}$ ALHAT HDS Software.

$\ddagger$ ALHAT NDL Signal Processing Lead.
} 
Vertical TestBed (VTB) $4[5 \mid 6$ Incorporating ALHAT capabilities onboard Morpheus or any VTB or spaceflight vehicle requires a systems-level perspective throughout the design, implementation and testing cycle of both the components and the integrated system. The focus of this paper is on the systems-level interfacing and functional verification of the ALHAT sensors as both individual units and as components integrated into a vehicle GNC subsystem. This paper is a companion to another recent paper on the preparation and integration activities of the ALHAT sensors and Morpheus GNC leading up to the joint flight testing! 5

The development of the ALHAT suite of capabilities has been through a multi-center collaboration within NASA, including NASA Johnson space Center (JSC), NASA Jet Propulsion Laboratory (JPLD), NASA Langley Research Center ( $(\overline{\text { LaRC}})$, and many other supporting contractors and research centers, including the Charles Stark Draper Laboratory (CSDL) and the Johns Hopkins Applied Physics Laboratory (APL). The ALHAT sensors shown in Figure 1 include a three-beam Navigation Doppler Lidar (NDL) ${ }^{7}$ that provides precise velocity measurements, a long-range Laser Altimeter (LAlt),$\frac{8}{8}$ and a flash Light detection And ranging (Lidar)-based ${ }^{\sqrt{9}}$ Hazard Detection System $(\underline{\mathrm{HDS}})^{10} 11$ that provides real-time Hazard Detection $(\underline{\mathrm{HD}})^{12}$ for onboard determination of safe landing sites. The Morpheus flight test performance of the individual sensors is highlighted in multiple recent papers (add SciTech citations).
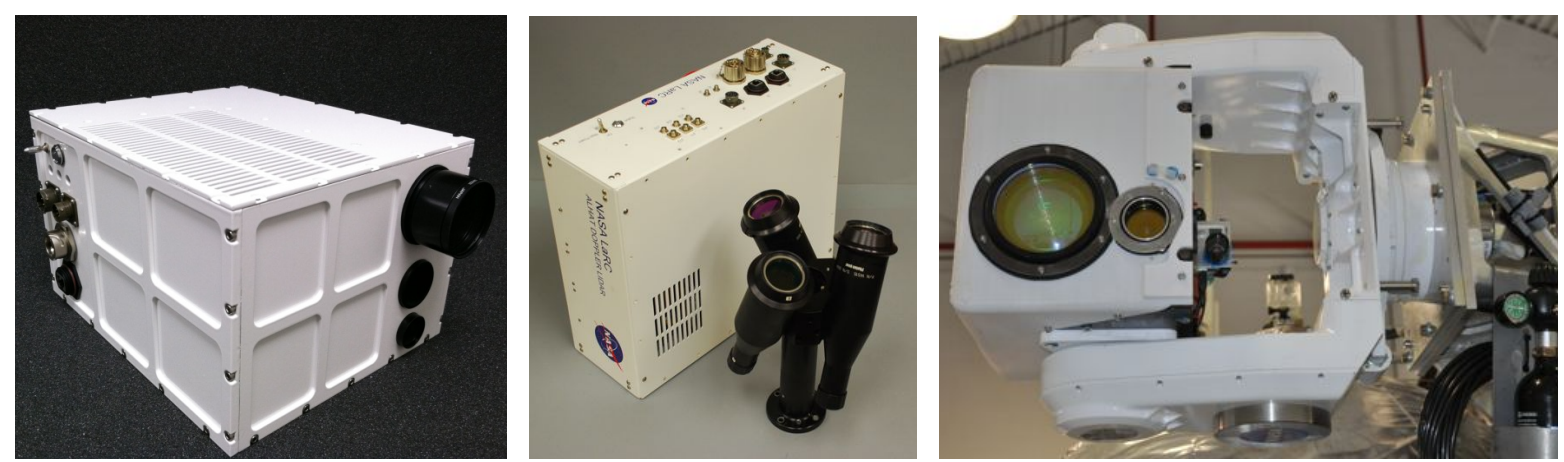

Figure 1. Prototype ALHAT sensors flight tested on Morpheus in 2014: long-range LAlt (left), NDL optics head and electronics (center), and HDS gimbaled flash Lidar (right).

The Morpheus vehicle was designed as an autonomous VTB for accelerating the development and infusion of new technologies into spaceflight applications. For Entry, Descent and Landing (EDL)-related GNC technologies, $\mathrm{VTB}$; provide the capability to test and refine at the integrated-system level in dynamicallyrelevant, closed-loop operation prior to launch and actual mission EDL For GNC development, the Morpheus Flight Software (FSW) includes a dual-string Navigation (Nav) architecture (Ref Jake paper) with a baseline VTB Nav filter and an experimental (in this case ALHAT) Nav filter. As the prototype GNC technology matures from initial open-loop tests into fully closed-loop tests, the VTB Nav will continue to run as a backup in case the prototype GNC performs outside of specified tolerances. The nominal VTB Nav estimates vehicle state from onboard Global Positioning System (GPS) measurements, along Inertial Measurement Unit (IMU) measurements from a Space Integrated GPS/Inertial Navigation System (SIGI) sensor and altimetry measurements from an Acuity range sensor. The experimental Nav string is configured to execute ALHAT Nav for the joint ALHAT/Morpheus flight testings, which utilizes only the ALHAT sensors and SIGI for in-flight measurements. The in-flight monitoring of ALHAT Nav is performed by an Autonomous Flight Manager (AFM) running within Morpheus FSW.

The culmination of ALHAT field tests, $\frac{10 \mid 13}{14}$ along with the closed-loop testing of ALHAT onboard Morpheus has operationally demonstrated the ALHAT techniques and approaches in a relevant flight-like environment, which raises the ALHAT Technology Readiness Level (TRL) to 6 and prepares it for infusion into future spaceflight applications. The remainder of this paper will provide some insight into the abundance of stand-alone and integrated development and test activities that have been a part of the flight testing and TRL advancement for ALHAT. Section II will give a short overview of the flight trajectories flown onboard Morpheus. The internal interfacing of ALHAT systems and the interfacing of ALHAT with the Morpheus avionics is discussed within Section III] This includes the definitions for data commanding and telemetry interfaces, as well as the architecture of sensor and vehicle time stamping. The testing and calibration 
activities for ALHAT as a vehicle subsystem are discussed in Section IV] Section $\mathrm{V}$ provides an overview of the functional verification tests that were conducted for the fully integrated ALHAT/Morpheus in preparation for flight tests, and closing remarks are provided in Section VI.

\section{Overview of Flight Profile}

Terrestrial flight testing of the ALHAT systems onboard Morpheus was conducted over a lunar-like terrain field constructed on the North end of the Shuttle Landing Facility (SLF) runway at Kennedy Space Center (KSC). The Morpheus flight profile and the phases of operation for the ALHAT sensors used within the ALHAT Nav filter during open-loop and closed-loop flight tests are illustrated in Figure 2. The flight profile is designed to put the vehicle in a dynamic state, following pitch over at peak altitude, that reproduces the conditions of a lunar approach for the Hazard Detection and Avoidance (HDA phase of ALHAT operation. The position and attitude of ALHAT Nav were initialized on the ground for the launch configuration, and during flight the only sensors providing measurement data to ALHAT Nav were the vehicle IMU and the three ALHAT sensors: LAlt, NDL and HDS. The LAlt provides altitude measurements to ALHAT Nav from 10 meters altitude on ascent until $\sim 25$ meters altitude on terminal descent. The HDS operations commence at a slant range of 450 meters and continue through a slant range of 200 meters; these operations include a HDA and Hazard Relative Navigation ( $\overline{\mathrm{HRN}}$ ) phase to generate the safe landing site and then provide siterelative position measurements, respectively. The $\mathrm{HDA}$ phase involves Digital Elevation Model/Map (DEM) generation followed by $\mathrm{HD}$ to determine the top safe landing site that the vehicle uses to conduct a hazard avoidance divert toward the safe location. The NDL provides velocimetry measurements following the completion of HDSIDEM generation until $\sim 25$ meters altitude on terminal descent. The IMU remains active from takeoff through touchdown so that the final $\sim 25$ meters are ALHAT Nav dead reckoning on the IMU.

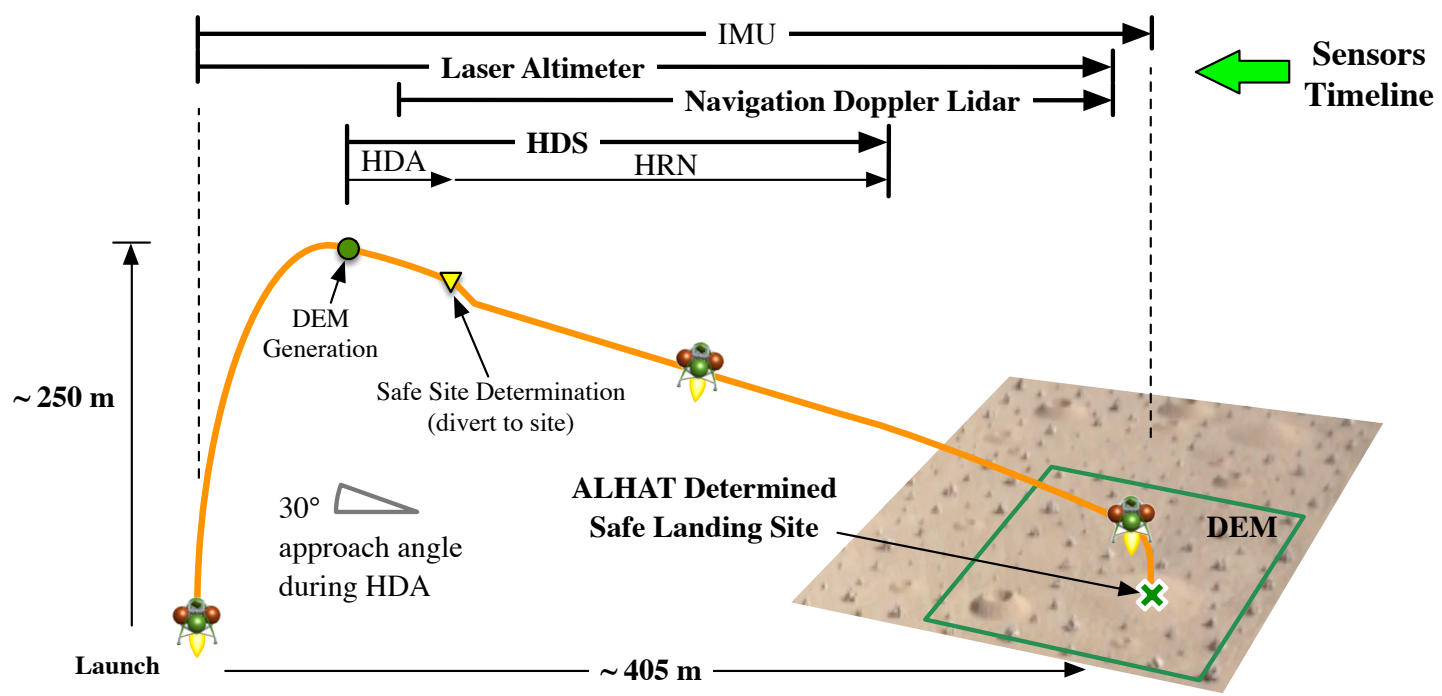

Figure 2. Flight profile and ALHAT sensors operational timeline during open-loop and closed-loop flight testing on Morpheus.

\section{System Interfacing}

With the ALHAT and Morpheus team spread across multiple NASA centers, the coordination of interfaces and integration activities between the ALHAT components and the Morpheus avionics was implemented through the development and implementation of ICD. The ICDs!s (ICDs!s) provides a set of specifications for interfacing not only mechanical hardware and electronics, but also provided guidelines for the different 
system elements to develop internal timing architectures, develop software interfaces for commanding and telemetry, and create visual interfaces for test and flight operations.

\section{III.A. Mechanical and Electrical Interfaces}

The performance of ALHAT is driven by the accuracy and precision of the individual sensor measurements, the formulation of the algorithms within the ALHAT Nav filter to process the measurements, and the knowledge of sensor hardware integration onto the Morpheus vehicle. The sensors and Nav algorithms can be developed and tested stand-alone, but the integrated performance is heavily influenced by knowledge of the physical integration. The mechanical ICD; between ALHAT sensors and the Morpheus structural mounts included details on bolt patterns and torque specifications, along with provisions for alignment features, such as pin/slot combinations and alignment edges. These features provided mechanical means for repeated alignments; when components were mechanically mounted, they were clocked against the alignment features in the direction of bolt tightening, which minimizes re-mount alignment differences.

The wiring and power interfaces were also outlined in ICDs that specified connector type, wire gauge, and intended purpose. All ALHAT intra-connect cables were developed, implemented and delivered from the ALHAT team, whereas all inter-connect cables between ALHAT and Morpheus components were codeveloped. For the inter-connect cables, the ALHAT sensor provider assembled the core cable bundle and the connector on the sensor side; the vehicle side of the cable was left pigtailed with long-lead wires. The vehicle wiring team then terminated the ALHAT cables according to the ICD once the ALHAT sensors were integrated onto the vehicle and the interface cables optimally routed base on accessibility and mass-reduction considerations.

\section{III.B. Managing Time}

Managing system time between the Morpheus VTB and the ALHAT sensors is critical for enabling precision landing capabilities. Relative timing errors between the vehicle time and the individual GNC sensor and ALHAT sensor times manifest as onboard Navigation errors in position, velocity and attitude. This in turn impacts onboard guidance algorithms that plan the descent profile to reach the desired safe landing site based on the knowledge provided from Navigation. The timing architecture within any synchronized system has two important attributes: 1) a reliable single source clock with reliable delivery method, and 2) an agreed upon interface to synchronize all other system endpoint clocks. The Morpheus VTB provides the central clock and delivery method to the ALHAT sensors, and a timing ICD specifies the synchronization method that is implemented between Morpheus and the ALHAT sensors.

The timing precision necessary for the terrestrial testing of Morpheus and ALHAT could be achieved with a typical GPS timestamp and Pulse Per Second (PPS) output provided from the Morpheus GPS receiver. The PPS signal occurs with each whole second rollover in GPS time. This time pulse provided the heartbeat for synchronizing the ALHAT sensors with Morpheus time. Distribution of the heartbeat can be accomplished by means as simple as using a splitter to distribute the clock rising edge or as complex as a digital phase relay with parallel processing and byzantine resilient voted output. The Morpheus VTB implemented the former method, being designed primarily as a zero fault tolerant system for simplicity and cost savings in developing the terrestrial testbed.

For the Morpheus time initialization, the flight computer on initial boot up waits for a valid GPS timestamp followed by three valid PPS pulses. Once received and checked for validity, the flight computer time is set to the GPS timestamp and then adjusted to the PPS whole second. The adjustment is essential in that the GPS timestamp in Morpheus occurs at $5 \mathrm{~Hz}$ and is not guaranteed to arrive at a 1-second boundary. The PPS, however, is "guaranteed" to appear at the 1-second boundary. The clock corrects itself with each PPS signal arrival (sometimes forward, sometimes backward) with the adjustment typically made in the microsecond resolution. The adjustment is only only allowable within a small window around the 1-second threshold to protect against any stray signal on the wire (e.g., Electro-Magnetic Interference (EMI) ) being interpreted as the PPS signal. 
The time ICD between Morpheus and ALHAT requires the delivery of a PPS heartbeat that is immediately followed (prior to the next $\overline{\text { PPS }}$ ) by a separate ethernet packet containing the Morpheus vehicle time defined in "seconds since epoch" of a pre-defined epoch. The ALHAT sensors synchronize to Morpheus vehicle time by latching whole seconds to the PPS, The handling of sub-seconds is done internally to each of the ALHAT sensors with slight implementation variations, but effectively the $\mathrm{PPS}$ arrival signals a roll over to the next whole second.

The HDS in particular has a further layer of complexity with time handling. The HDS generation of safe landing sites during $\mathrm{HD}$ and site-relative position measurements during $\mathrm{HRN}$ requires the generation of a seamless DEM and knowledge of image times relative to the Morpheus Nav state. This process requires the real-time fusion of multiple, asynchronous sensors internal to the HDS. To enable this fusion, the HDS Compute Element (HDSCE) design incorporates a Field Programmable Gate Array (FPGA) for internal time management, to aggregate all Input/Output (I/O) data, and to annotate the $\mathrm{I} / \mathrm{O}$ data with a system time associated with a measurement event from each internal sensor.16 This implementation both reduces I/O requirements and eliminates tight real-time Operating System requirements for the HDSCE processor. Additionally, having a single central system clock within the HDS avoids the need to manage different time interpretations across the many different internal-HDS sensors (gimbal, IMU, Lidar). Each of these internal devices provide a discrete event signal flag that indicates to the FPGA when the device is taking a measurement. When data for that device flows through the FPGA, its data packet gets annotated with the contents of the "event time" register and passed to the HDSCE processor for use in DEM generation, HD or HRN processing.

\section{III.C. Software Interfaces: Commanding and Telemetry}

The Morpheus andALHAT teams were distributed across multiple NASA centers, and individual components were developed seperately and not physically connected until very late in the design cycle. This made defining the ICD between the various components critical, as there would be limited time available for debugging/troubleshooting. To ensure all the components would talk with each other (and minimize schedule impacts from any integration troubleshooting ) we developed a software ICD for each of the ALHAT sensors (NDL/LAlt,HDS) and the Morpheus Flight computer (Avionics and Power Unit (APU)). Once adopted, these ICD s were only changed after agreement from all teams. So each team knew exactly what to send and receive from the others, and ths enabled interface checkouts prior to completion of the other components.

After obtaining consensus on the commands/telemetry in the ICD, the team then deployed a "software emulator" that could send an arbitrary data message to/from each sensor and Morpheus. These telemetry emulators were invaluable in ensuring that the Morpheus vehicle could send/receive messages to the sensors and helped catch interface issues (such as byte ordering, structure alignment, bit packing) prior to physical hardware integration. As a result we had a smooth SW interface integration every time the units were connected. The one thing that these emulators did not accurately replicate the precise timing of when messages would be sent; as noted in Section $\mathrm{V}$, these subtle timing variations caused some software issues that were not identified/addressed until integrated functional checkout.

The downlink telemetry link represented less than $2 \%$ of the data produced by Morpheus, which was logged onboard the vehicle. In contrast to the high bandwith data pipes available during the development process, once the vehicle was flying, the narrow (5KByte/second) telemetry link on Morpheus forced careful selection of which items to downlink. Selecting which parameters would be included in the downlink stream was a constant battle throughout the development process. This struggle required subsystem negotiation, and even resulted in a friendly contest (free dinner) to see which subsystem could reduce their bandwidth the most.

Once a set of parameters to downlink was agreed to, Morpheus utilized a "copy table" to set up a series of memory copies to extract the specified memory locations from various SW modules to produce a unified downlink message stream. This "copy table" needed to be synchronized with any ICD change of any onboard software modules. In addition, as the ground displays evolved, and operators needed to have access to new data items (or any increase/decrease the rate an item was sent) the copy table needed to be changed. And each new copy table needed to be verified to ensure it was error free and in sync with the ground software so 
it would be decoded correctly. Generation of the copy table was initially done by hand, but it was quickly apparent that as we needed an automated process to make verification of the synchronization a feasible task since even a minuscule change to the flight SW (say 1 additional byte of status data) could trigger hundreds of changes in the copy table, and would take hours of time to do (and verify) by hand!

Eventually, the Command And Data Dictionary (CDD) for Morpheus was established as the authoritative version control system for the "copy table" and Integrated Test and Operations System (ITOS) rec files. The CDD also contained the limits (high and low) for parameter values, downlink frequency, and the calibration values for each parameter. After building a second APU, it became apparent that the analog channels on the two systems were calibrated differently enough that separate sets of calibration constants was needed for to each APU. The correct constants were obtained by specifying which APU was being targeted when running the CDD auto-generation software toolchain.

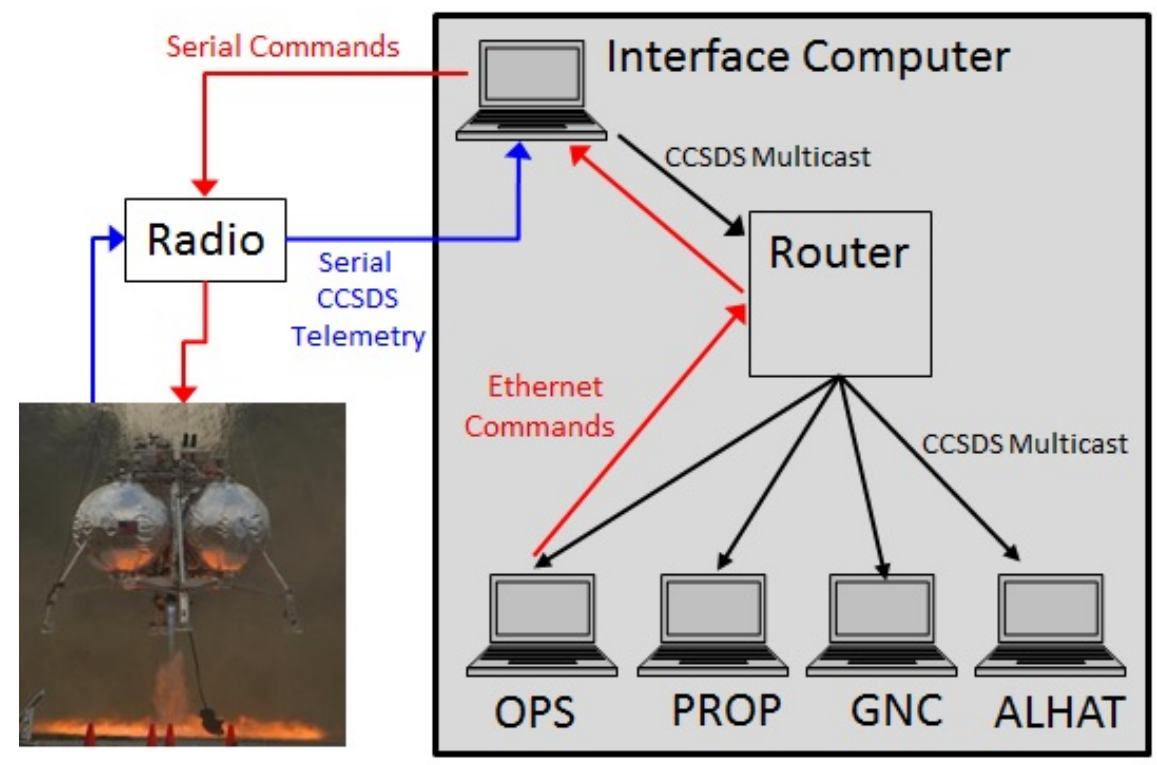

Figure 3. Command and Telemetry path for Morpheus ALHAT

\section{III.D. Operations Interfaces}

ITOS was used to perform all monitoring and control of the Morpheus vehicle while in flight 17 ITOS was developed by Goddard Space Flight Center (GSFC) to communicate with space vehicles using Core Flight Software (which is the software used in Morpheus). Since the ALHAT sensors data was integrated into the vehicle telemetry (See Section III.C), the Morpheus data link contained ALHAT telemetry. The single data path to the Morpheus vehicle was connected to a "headless" interface computer, whose sole job was to relay to data from the control room to the vehicle. (See Figure 3 ).

Because all of the data from the vehicle was sent via multicast to the control room, each computer had equal access to all the telemetry, and could bring up any page/plot in ITOS. The single multicast of the data eliminated the need for a separate transmission to each of the computers in the control room: the same amount of work was done by the Interface Computer if there were 1 or 100 computers consuming the telemetry. This multicast data was also "reflected" to JPL/JSC/LaRC, so that ITOS data could be viewed remotely in real time across the country by team members who were not physically present at KSC for a test. Each console position would generally be monitoring only the ITOS pages/plots that were relevant to them during the tests. In fact, most of the pages for the various console positions were built by the subsystems, allowing them to see exactly the data they wanted.

Only a single station could send commands to the Morpheus vehicle. This was done partly to avoid command contention issues that could arise from allowing multiple computers to send commands, and also 
because the command processor in ITOS consumed a fair amount of CPU resources, and the computers were more responsive when it was not running. Any station could bring up the command pages, and "push" a command buttons, but no commands would be sent unless they originated from the Operator console station. This helped reduce the chance of issuing a command accidentally, since all commands would be ignored from most computers in the control room. A few examples of the hundreds of pages/plots developed for Morpheus, ALHAT can be see in Figure 4.

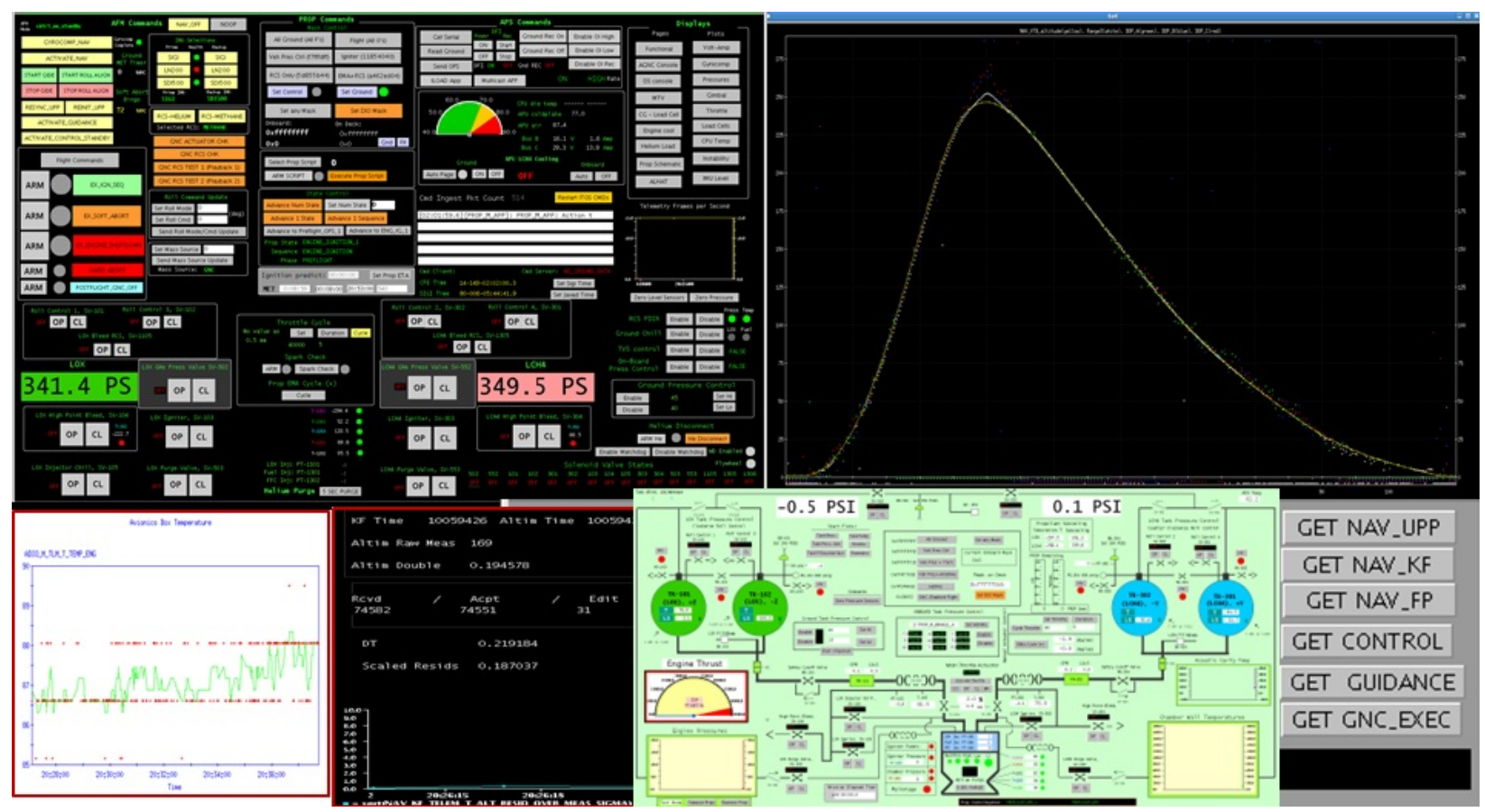

Figure 4. Sample of some of the pages and plots used to monitor Morpheus ALHAT

\section{ALHAT Testing prior to Morpheus Vehicle Integration}

The flight performance of ALHAT onboard Morpheus is driven by both the stand-alone ALHAT sensor performance and the integrated performance of ALHAT with the Morpheus GNC subsystem. Prior to the flight testing at KSC, the ALHAT project conducted multiple, stand-alone field and calibration tests, $10,18,19$ along with several joint tests with Morpheus avionics $11|13| 14$ and flight simulations. These tests were important for the development and refinement of ALHAT technologies, as well as for risk mitigation leading up to the flight testing on Morpheus. This section highlights some of the calibration activities, as well as field testing and simulations conducted while maturing ALHAT and preparing it for the Morpheus flight tests.

The NDL sensor provides three individual Line of Sight (LOS) velocity measurements that are used to resolve the Morpheus velocity vector. Calibration of the LOS measurements for each beam, along with knowledge of each beam direction relative to the NDL mechanical assembly is essential for resolving the spacecraft velocity vector in the body frame. The beam calibrations were established by comparing LOS measurements with independently measured motion of a high-fidelity and very-low-distortion sub-woofer driven at low frequency. The alignment knowledge for each beam was established through metrology measurements shown in Figure 5 of point targets along each beam LOS relative to fiducial locations on the mechanical base onto which each telescope attaches.

The HDS in-flight performance depends on how well the sensor can point the flash-Lidar optical boresight vector to obtain the desired terrain images for HD and HRN. The pointing performance is affected by component-to-component alignment and calibration knowledge within the HDS, as well as the accuracy 

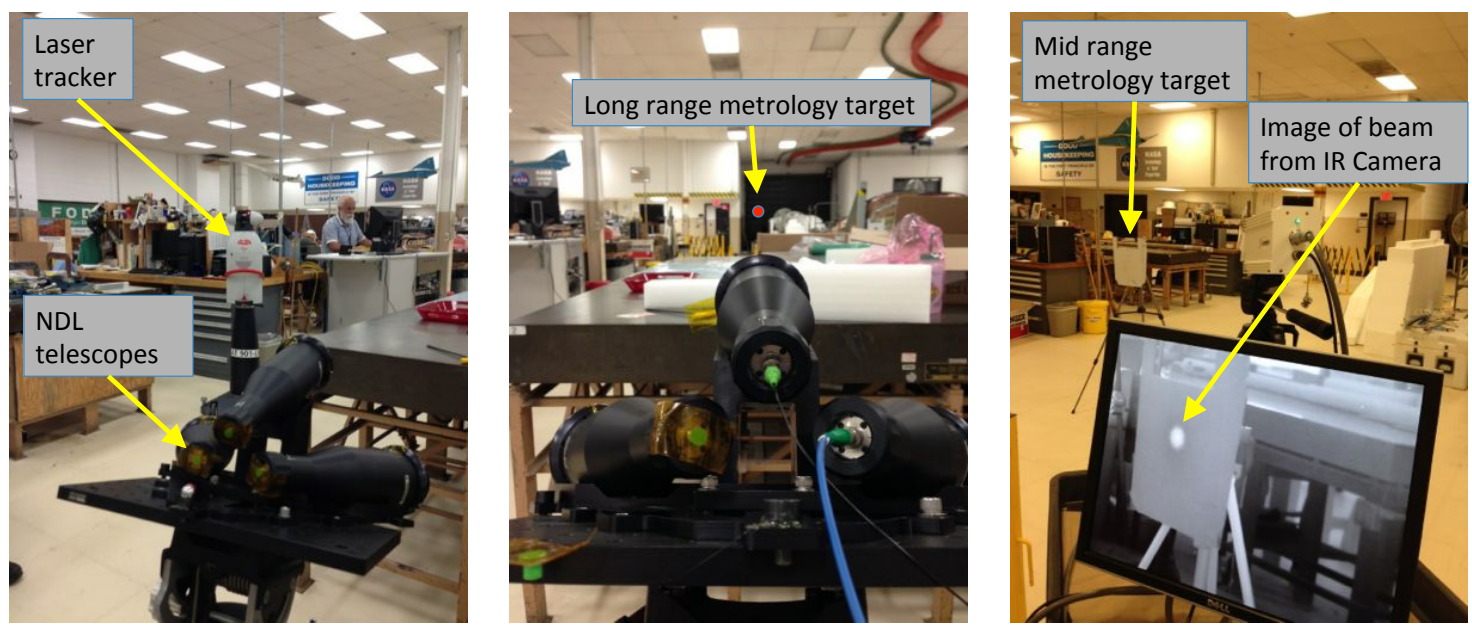

Figure 5. Metrology measurements of NDL Line-of-Sight beams relative to mechanical mounting fixture.

of the ALHAT Nav estimate that HDS uses for pointing toward a desired terrain region. To establish internal component-wise alignment, a laser metrology system was used to resolve the FL boresight vector relative to the FL mechanical mount, the FL mechanical mount relative to the inner gimbal rotation axis, the rotation vectors for both gimbal axes, and the gimbal mount relative to the HDS IMU. Additionally, long-distance range calibrations were performed to determine the accuracy and precision of the FL sensing, and 19-bit encoders were used for measuring gimbal pointing angles to minize error. To improve further on the component-wise alignment and calibration, an end-to-end pointing test to multiple distant targets (also measured with metrology) was performed, and the offset errors were averaged to provide a final end-to-end correction for systematic bias. The end result is a stand-alone HDS with pointing knowledge to within $0.05^{\circ}$ of the commanded target. For further information on the HDS alignment and calibration processes, along with details on general HDS operations or other field testing, refer to the references 11$] 13$

Alignment of the HDS to ALHAT Nav is of critical importance for HD and HRN during flight tests. To assemble the DEM for HD, the HDS gimbal slews the FL to collect a mosaic of terrain images that are seamlessly fused together in software. To avoid the potential for holes in the DEM, the HDS does not accept Nav updates during mosaicking but instead propagates the Nav solution with the dedicated HDS IMU. To minimize pointing error growth during this propagation, the relative attitude between the HDS IMU and the Morpheus SIGI was obtained through analysis of long-dwell data collected in multiple vehicle tilt configurations (Figure 6).
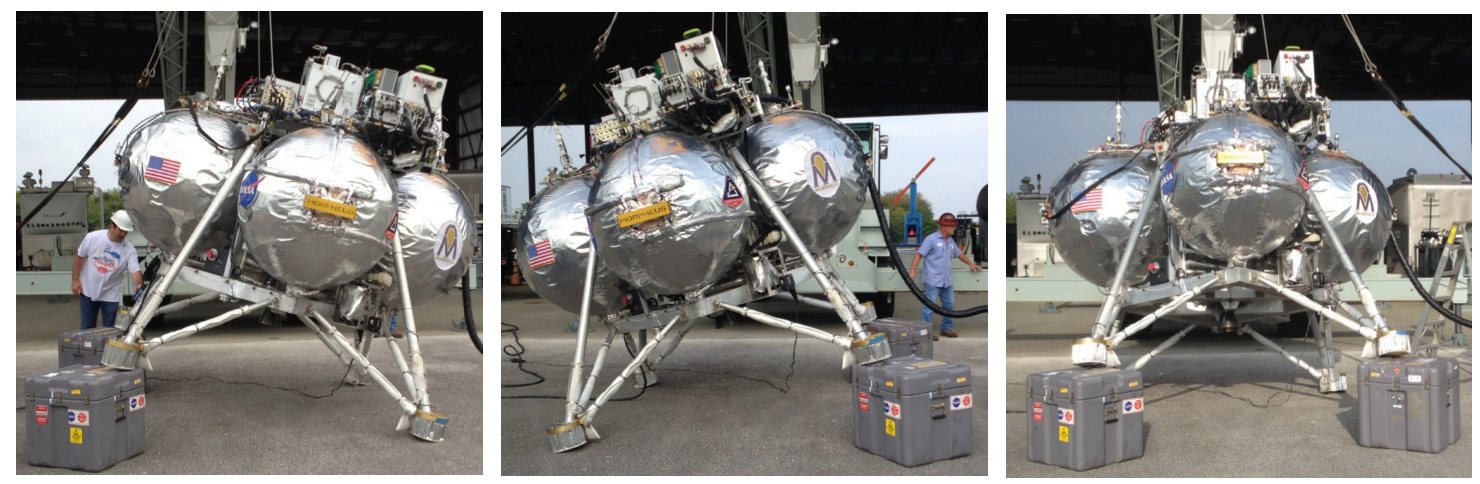

Figure 6. Tilt tests of integrated Morpheus vehicle with ALHATHazard Detection System to compare gyro orientations with metrology measurements.

The HDS Flash Lidar (FL) has a narrow $1^{\circ}$ Field of View (FOV) lens, which was chosen so that the 
HDS could created 10-cm resolution DEM from up to a 1-kilometer range. The narrow FOV provides some challenges in situational awareness when post processing the FL data to resolve pointing errors or data ambiguities. For this reason, an optical witness camera was mounted under the FL sensor head and co-aligned with the FL boresight (Figure 7 ) to provide a larger FOV image ( $8^{\circ}$ along the diagonal). The witness camera is aligned to the HDS FL by pointing the HDS to two distant targets and assessing the relative parallax between the FL and witness camera images. The same two targets are also used for assessing Nav initialization that will be discussed in Section $\mathrm{V}$.

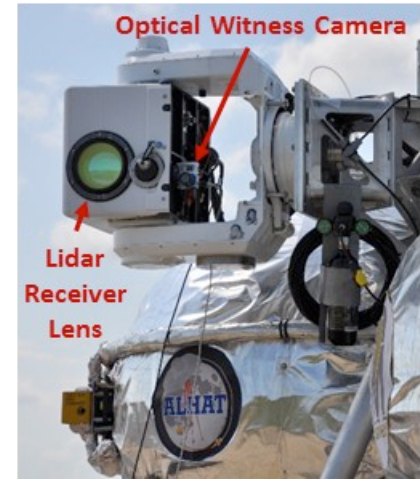

Figure 7. An optical witness camera with larger FOV] that the HDS FL provides additional situational awareness in post-flight data analysis and in pre-flight checkouts.

Early functional interfacing and integrated testing between ALHAT and Morpheus avionics (primary flight computer and SIGI) were conducted in labs and in two field tests. The lab tests were primarily for interfacing components and were dubbed FlatSat testing since the avionics and sensors were tested on a flat bench. The field testing was conducted on two dynamic platforms, a truck and a helicoptel $15 \sqrt[13]{21}$ as seen in Figure 8 . These field tests were valuable for the development and functional testing of the ALHAT sensors interfaced with the Morpheus avionics and provided risk reduction for the joint free flight testing on the Morpheus VTB. The truck testing was conducted at the LaRC Long Distance Test Range (LDTR), which is of an 800+ meter test track with a target wall on one end. The ALHAT sensors and electronics were located within the cargo area of the truck as seen in figure. The HDS and LAlt had a forward view through a window in the front of the cargo hold, and the NDL was cantilevered off the rear of the truck with a ground view. The target was configured with hemispherical targets to allow functional testing of HDS DEM generation and HD algorithms, as well as pointing operations on the ALHAT Nav. The helicopter flight tests were conducted KSC over a lunar-like terrain field constructed for the Morpheus VTB flight test and provided the closest possible ALHAT operational data prior to the actual free flights on Morpheus.

\section{Truck Testing}
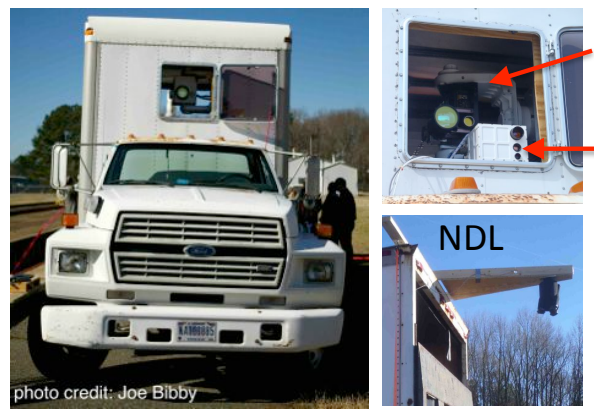

Helicopter Testing
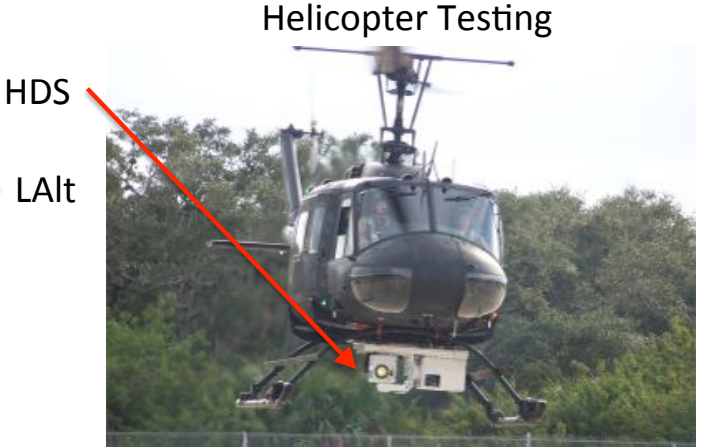

Figure 8. Joint field tests were conducted with ALHAT and Morpheus avionics on a truck (left) and helicopter (right) in preparation for free flight tests on the Morpheus vehicle.

In additional to early interfacing testing, simulations were used to understand and refine the Concept of Operations (ConOps) and integrated performance of ALHAT on Morpheus prior to integration and flight 
testing with the actual systems. The team conducted extensive testing using NASA's Trick ${ }^{20}$ simulation tool that runs the full Morpheus FSW set. The Trick simulation framework (developed by NASA/JSC) provides a full 6-DOF environmental simulation, and is currently in use by a wide range NASA projects. In addition to using the flight software, Trick requires a model for each sensor, as well as a models for each actuator that can be controlled by the vehicle. The simulator sensor models construct measurements based on the simulated translation and rotation state of the vehicle. These measurements are fed into the FSW] which processes the them and decides what commands to send to the actuators. Trick then evaluates the actuator models and calculates the next state of the vehicle (and then starts the loop again). The benefit to using this framework is to determine what the flight code would do in response to given stimulus without risking the vehicle, as well as to allow running Monte Carlo simulations to see how sensitive the outputs are to various parameters.

\section{Functional Verification onboard Morpheus for Flight Tests}

Functional verification of the ALHAT sensors with the Morpheus GNC and Avionics subsystems was necessary following integration of ALHAT onto Morpheus. The verifications initially included whole-system power up and command/telemetry checks between the Morpheus flight operations displays and the ALHAT sensors. More focused tests looked at the individual ALHAT sensor measurements to verify good time synchronization and proper incorporation of alignment information for Nav processing of the measurements. The verifications concluded with tether testing of the fully integrated vehicle prior to free flights, followed by health and status checks of the ALHAT sensors in between flights.

A functional verification of ALHAT Nav integration with the LAlt and NDL was performed through a swing test of the entire Morpheus vehicle suspended on a crane, as shown in Figure 9. The low altitude and oscillation rate of the swing test put the NDL in a mode of operation outside of the design implementation, which precluded precision Navigation testing, but nonetheless, the test provide a desired functional verification of good time synchronization and proper incorporation of metrology alignment transforms. Comparison of the VTB Nav (based on GPS, SIGI and Acuity measurements) estimates for velocity with the direct NDL measurements of velocity verified alignment transforms. The comparisons also revealed any time lags due to system initialization or unresolved integration bugs. The lifting and lowering of the vehicle for the swing test also allowed direct comparisons between the Acuity range measurement and the LAlt measurements, which were used for basic calibration checks. Initial swing tests revealed a polarity flip in how ALHAT Nav handled the NDL LOS velocity measurements and also helped the ALHAT and Morpheus teams refine operational procedures for flight testing.
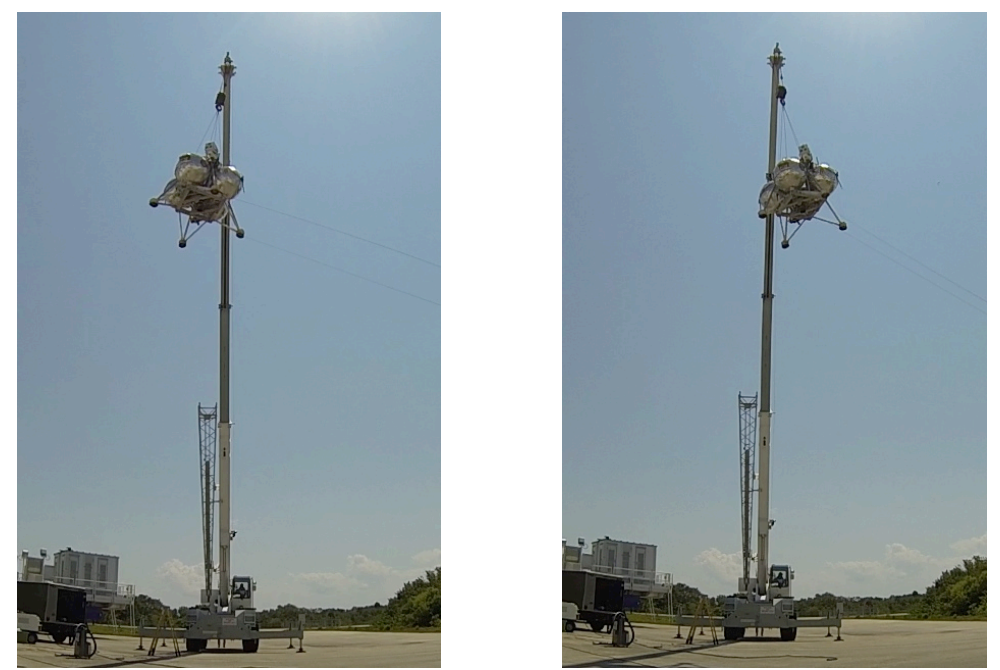

Figure 9. Swing tests of integrated Morpheus vehicle with ALHAT Navigation Doppler Lidar and Laser Altimeter. 
Throughout the campaigns, the HDS FL pixels were monitored for degradation because the prototype sensor is not hermetically sealed. Pixel damage can be caused from adverse environmental conditions (mainly conditions of either high relative humidity or dew point temperatures near the focal plane array's operating temperature) or focal plane array age. In between flights and during all pre-flight operations, the FL is purged with dry air to minimize humidity- and dew point-related damage. An in-flight purge was not in place for early flights, so pixel damage was assessed between flights. Intermittent, damaged pixels generally produce range solutions between zero meters and range values within one to two meters of the actual range value of the surrounding pixels, which makes them them difficult to filter. The damaged pixels can cause holes or spikes in the DEM that are interpreted as hazards, especially if the damaged pixels cluster together. If the bad pixels are known prior to flight, the problematic regions can be masked out in the process of DEM generation. Prior to each flight, on-ground testing was conducted to determine if the Flash Lidar?s pixel mask needed to be expanded to cover any new intermittent pixels. The on-ground testing involves imaging a flat target board at a near zero degree angle of incidence so that all of the 16,384 pixels should produce the same range solution within the precision limit of the sensor. Any pixels which produce range values outside the precision limit are flagged as intermittent pixels and are added to the pixel mask which assigns a range value of zero to them so that they can be easily filtered from the range image during real-time processing. Figure 10 shows a pixel mask test. In the test, the FL median filter has been temporarily deactivated so that individually masked pixels are visible and display zero range. Several new intermittent pixels are also visible; they produce range values approximately 1 meter in error and need to be added to the FL pixel mask.

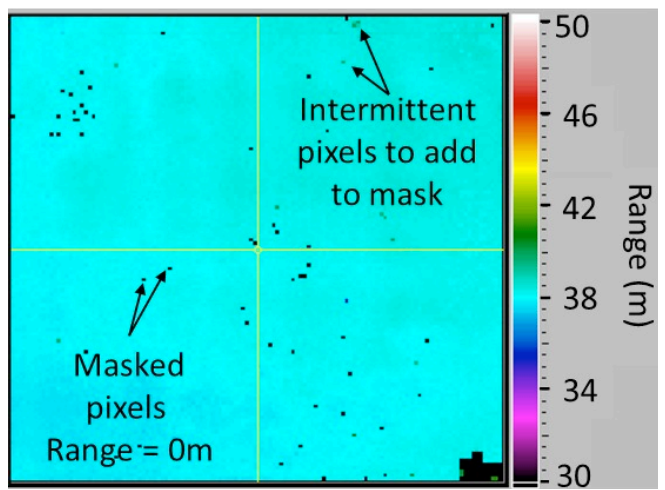

Figure 10. Flash Lidar pixel mask test (with median filter temporarily deactivated) from $5 / 20 / 2014$ in which the flat GIDE target board is imaged at approximately a zero degree incidence revealing currently masked pixels with zero range and intermittent pixels which need to be added to the mask.

The tight coupling of HDS performance with the onboard Nav accuracy drove the inclusion of a groundbased HDS pointing test to verify Nav initialization prior to every flight test. The operational design for HDS requires combined pointing knowledge error between HDS and Nav to be less than $0.25^{\circ}$. The onboard VTB and ALHAT Nav states are initialized on the ground through a process of gyrocompassing and roll alignment to determine the initial inertial position and attitude of the vehicle; an optical sextant mounted on the vehicle is used to measure angular offsets to a known, surveyed ground target and determine roll (yaw in aircraft convention) corrections to improve on the gyrocompassed attitude solution. Following Nav initialization, the HDS is commanded to point the FL at two GPS-surveyed ground targets (Figure 11) at approximately 88 and 349 meter ranges. The full FL range image is not sent through telemetry to the Morpheus mission control center due to vehicle communications bandwidth limitations, but an average range for the central nine pixels is transmitted, along with an optical image from the witness camera discussed in Section IV] The witness camera image is observed in the control room, along with the range reported from the FL telemetry data, to assess whether or not the initial Nav solution provides the accuracy required for pointing HDS.

The final full-up functional checkout prior to free flight testing is a tether test (Figure 12) of the ALHAT systems onboard Morpheus. After every major change to the vehicle, or after extended downtime, the team 

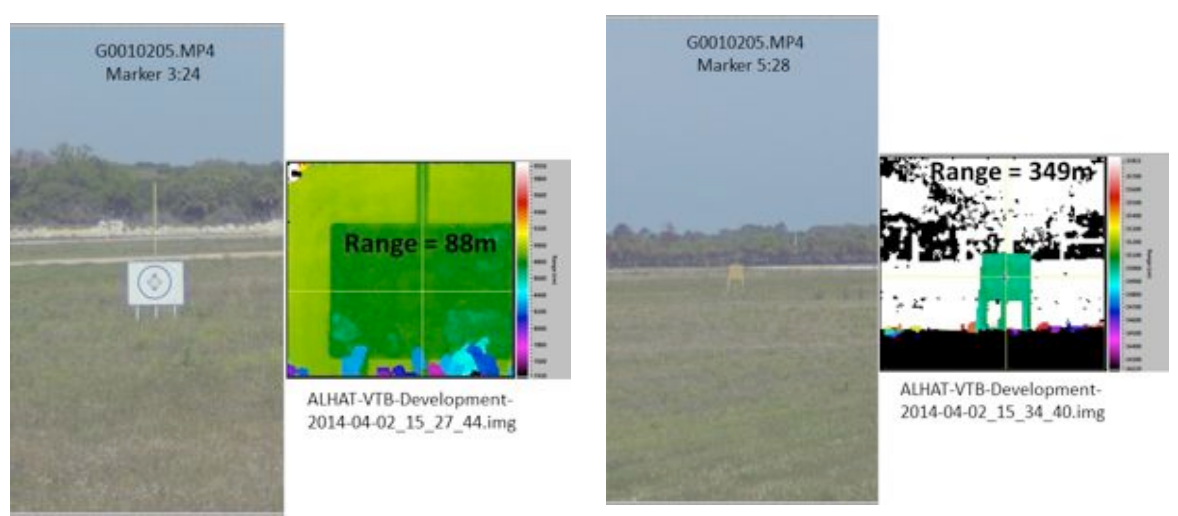

Figure 11. HDS pointing to near (left) and far (right) surveyed targets based on Nav initialization: Co-boresighted witness camera images and FL range images are shown.

performed a tethered flight test to verify function and operation. For the ALHAT sensors, the tethered flights are primarily a test of in-flight function rather that an opportunity to collect valid data for performance analysis. The low altitude and minimal surface-relative speeds limit the valid data coming from the LAlt and NDL. Additionally, the shallow viewing angle and short range to the ground causes the HDS FL to de-focus and not provide valid terrain images. Nonetheless, the tethered flights provide and opportunity for integrated functional opperation between ALHAT and the GNC subsystem. Early tethered flights were used for initial verification of dynamically pointing the HDS from the onboard Nav state. These tests helped to identify time lags within the system between the Nav state sent to the HDS and the higher-rate HDS IMU.

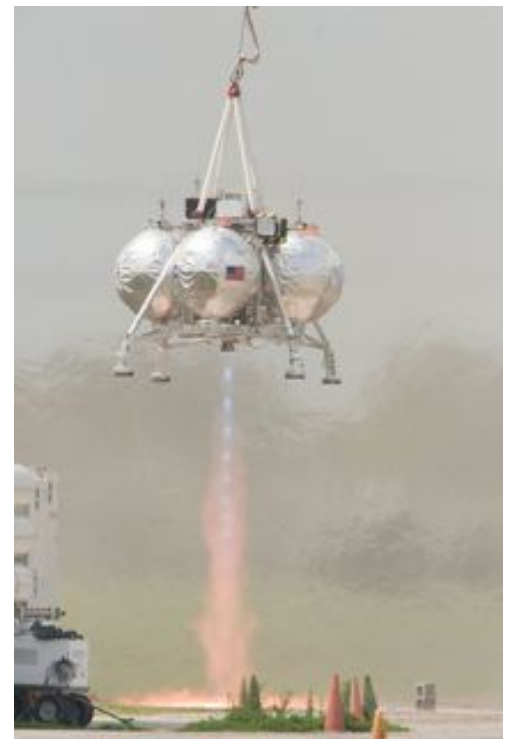

Figure 12. Tether flight of ALHAT sensors in preparation for free flight testing.

\section{Closing Remarks}

The successful integrated performance of the ALHAT sensors onboard Morpheus during the 2014 joint flight testing was made possible through the systems engineering of the ALHAT sensors, the Morpheus vehicle, and the collaborative approach to integration and testing. The detailed development, calibration and testing of the stand-alone ALHAT sensors provided known precision and accuracy during sensor operation. 
The use of well-defined interfaces between the sensors and vehicle, along with the detailed integration testing and follow-ion functional verifications provided confidence that the ALHAT and Morpheus systems would perform as desired in free flight tests. The free flight tests in 2014 were highly successful, providing a wealth of data for analyzing and refining ALHAT sensors performance and for raising the overall TRL of the ALHAT systems to a 6 , preparing it for infusion into future spaceflight applications.

\section{List of Acronyms}

AFM Autonomous Flight Manager

ALHAT Autonomous precision Landing and Hazard Avoidance Technology

APL Johns Hopkins Applied Physics Laboratory

APU Avionics and Power Unit

CDD Command And Data Dictionary

ConOps Concept of Operations

CSDL Charles Stark Draper Laboratory

DEM Digital Elevation Model/Map

EDL Entry, Descent and Landing

EMI Electro-Magnetic Interference

FL Flash Lidar

FOV Field of View

FPGA Field Programmable Gate Array

FSW Flight Software

GNC Guidance, Navigation and Control

GPS Global Positioning System

GSFC Goddard Space Flight Center

HD Hazard Detection

HDA Hazard Detection and Avoidance

HDS Hazard Detection System
HDSCE HDS Compute Element

HRN Hazard Relative Navigation

ICD Interface Control Design/Document

ITOS Integrated Test and Operations System

I/O Input/Output

IMU Inertial Measurement Unit

JPL Jet Propulsion Laboratory

JSC Johnson space Center

KSC Kennedy Space Center

LAlt Laser Altimeter

LaRC Langley Research Center

LDTR Long Distance Test Range

Lidar Light detection And ranging

LOS Line of Sight

Nav Navigation

NDL Navigation Doppler Lidar

PPS Pulse Per Second

SIGI Space Integrated GPS/Inertial Navigation System

TRL Technology Readiness Level

VTB Vertical TestBed

\section{References}

\footnotetext{
${ }^{1}$ Epp, C. and Smith, T., "The Autonomous Precision Landing and Hazard Detection and Avoidance Technology (ALHAT)," Proc. Space Technology and Applications International Forum (STAIF), 2007.

${ }^{2}$ Epp, C., Robertson, E., and Brady, T., "Autonomous Landing and Hazard Avoidance Technology (ALHAT)," Proc. IEEE Aerospace Conference (AEROCONF 2008), March 2008.

${ }^{3}$ Brady, T., Schwartz, J., and Tillier, C., "System Architecture and Operational Concept for an Autonomous Precision Lunar Landing System," AAS 30th Rocky Mountain Guidance and Control Conference, February 2007.

${ }^{4}$ Epp, C. D., Robertson, E. A., and Carson III, J. M., "Real-Time Hazard Detection and Avoidance Demonstration for a Planetary Lander," Proc. AIAA SPACE 2014 Conference 6 Exposition, San Diego, CA, Aug. 5-7 2014.
} 
${ }^{5}$ Carson III, J. M., Robertson, E. A., Pierrottet, D. F., Roback, V. E., Trawny, N., Devolites, J. L., Hart, J. J., Estes, J. N., and Gaddis, G. S., "Preparation and Integration of ALHAT Precision Landing Technology for Morpheus Flight Testing," Proc. AIAA SPACE 2014 Conference 83 Exposition, San Diego, CA, Aug. 5-7 2014.

${ }^{6}$ Olansen, J. B., Munday, S. R., and Devolites, J. L., "Project Morpheus: Lander Technology Development," Proc. AIAA SPACE 2014 Conference ES Exposition, San Diego, CA, Aug. 5-7 2014.

${ }^{7}$ Amzajerdian, F., Pierrottet, D., Petway, L., Hines, G., and Barnes, B., "Doppler lidar sensor for precision navigation in GPS-deprived environment," Proc. International Society for Optics and Photonics (SPIE), June 2013.

${ }^{8}$ Pierrottet, D., Amzajerdian, F., and Barnes, B., "A long distance Laser Altimeter for terrain relative navigation and spacecraft landing," SPIE Defense and Security Symposium, June 2014.

${ }^{9}$ Amzajerdian, F., Pierrottet, D., Petway, L., Hines, G., and Roback, V., "Lidar systems for precision navigation and safe landing on Planetary Bodies," Proc. International Society for Optics and Photonics (SPIE), August 2011.

${ }^{10}$ Keim, J., Mobasser, S., Kuang, D., Cheng, Y., Ivanov, T., Johnson, A., Goldberg, H., Khanoyen, G., and Natzic, D., "Field test implementation to evaluate a flash LIDAR as a primary sensor for safe lunar landing," IEEE Aerospace Conference (AEROCONF 2010), March 2010.

${ }^{11}$ Carson, J. M., Bailey, E. S., Trawny, N., Johnson, A. E., Roback, V. E., Amzajerdian, F., and Werner, R. A., "Operations Concept, Hardware Implementation and Ground-Test Verification of a Hazard Detection System for Autonomous and Safe Precision Lunar Landing," Proc. AAS/AIAA Astrodynamics Specialist Conference, Hilton Head Island, SC, Aug. 11-15 2013.

${ }^{12}$ Ivanov, T., Huertas, A., and Carson, J., "Probabilistic Hazard Detection for Autonomous Safe Landing," Proc. AIAA Guidance, Navigation, and Control Conference, August 2013.

${ }^{13}$ Trawny, N., Carson, J. M., Huertas, A., Luna, M. E., Roback, V. E., Johnson, A. E., Martin, K. E., and Villalpando, C. Y., "Helicopter Flight Testing of a Real-Time Hazard Detection System for Safe Lunar Landing," Proc. AIAA SPACE 2013 Conference E Exposition, San Diego, CA, 10-12 Sept. 2013.

${ }^{14}$ Pierrottet, D. F., Amzajerdian, F., Petway, L. B., Hines, G. D., and Barnes, B., "Field Demonstration of a Precision Navigation Lidar System for Space Vehicles," Proc. AIAA Guidance, Navigation, and Control Conference, Boston, MA, August 2013.

${ }^{15}$ Rutishauser, D. K., Epp, C. D., and Robertson, E. A., "Free-Flight Terrestrial Rocket Lander Demonstration for NASA's Autonomous Landing and Hazard Avoidance Technology (ALHAT) System," Proc. AIAA SPACE 2012 Conference ES Exposition, September 2012.

${ }^{16}$ Villalpando, C., Werner, R., Carson III, J., Khanoyan, G., Stern, R., and Trawny, N., "A Hybrid FPGA/Tilera Compute Element for Autonomous Hazard Detection and Navigation," Proc. IEEE Aerospace Conference (AEROCONF 2013), March 2013.

${ }^{17}$ Pfarr, B., Donohue, J., Lui, B., Greer, G., and Green, T., "Proven and Robust Ground Support Systems - GSFC Success and Lessons Learned," Aerospace Conference, 2008 IEEE, March 2008, pp. 1-7.

${ }^{18}$ Johnson, A., Keim, J., and Ivanov, T., "Analysis of Flash Lidar Data Field Test Data for Safe Lunar Landing," Proc. IEEE Aerospace Conference (AEROCONF 2010), March 2010.

${ }^{19}$ Johnson, A. E., Keim, J., and Ivanov, T., "Analysis of Flash Lidar Data Field Test Data for Safe Lunar Landing," Proc. IEEE Aerospace Conference (AEROCONF 2010), March 2010.

${ }^{20}$ Paddock, E. J., Lin, A., Vetter, K., and Crues, E. Z., "Trick: A Simulation Development Toolkit," AIAA Modeling and Simulation Technologies Conference and Exhibit, 2003, March 2003.

${ }^{21}$ Roback, V. E., Bulyshev, A. E., Amzajerdian, F., Brewster, P. F., Barnes, B. W., Kempton, K. S., and Reisse, R. A., "Helicopter Flight Test of a Compact, Real-Time 3-D Flash Lidar for Imaging Hazardous Terrain during Planetary Landing," Proc. AIAA SPACE 2013 Conference 83 Exposition, San Diego, CA, 10-12 Sept. 2013. 